Tôhoku Math. Journ. 39 (1987), 27-40.

\title{
A CHARACTERIZATION OF THE CARTAN HYPERSURFACE IN A SPHERE
}

\author{
U-HANG KI* AND HISAO NAKAGAWA**
}

(Received January 20, 1986)

Introduction. Let $S^{n}(c)$ be an $n$-dimensional sphere of constant curvature $c$ in an $(n+1)$-dimensional Euclidean space $\boldsymbol{R}^{n+1}$. A hypersurface in $S^{4}=S^{4}(1)$ defined by the equation

$$
2 x_{5}^{3}+3\left(x_{1}^{2}+x_{2}^{2}\right) x_{5}-6\left(x_{3}^{2}+x_{4}^{2}\right) x_{5}+3 \times 3^{1 / 2}\left(x_{1}^{2}-x_{2}^{2}\right) x_{4}+6 \times 3^{1 / 2} x_{1} x_{2} x_{3}=2
$$

was investigated by E. Cartan [1], who proved that this space is a homogeneous Riemannian manifold $S O(3) /\left(\boldsymbol{Z}_{2} \times \boldsymbol{Z}_{2}\right)$ and that the principal curvatures of the hypersurface are equal to $3^{1 / 2}, 0$ and $-3^{1 / 2}$ everywhere. This is called the Cartan hypersurface in $S^{4}$, which is one of isoparametric hypersurfaces in a sphere. The classification of isoparametric hypersurfaces in a real space form has been studied by Münzner [8], Ozeki and Takeuchi [9], Takagi and Takahashi [15] and so on.

For the Cartan hypersurface in a sphere $S^{4}$, there seem to be two studies from different points of view: $n$-dimensional homogeneous hypersurfaces in a real space form were investigated by Kobayashi [5] and Takahashi [16], who gave the classification except when $n=3$ and type number 2, or when $n=2$. Takagi [14] noted that the exceptional case actually characterizes the Cartan hypersurface, i.e., the 3-dimensional Cartan hypersurface is the only connected homogeneous hypersurface in $S^{4}$ whose type number is equal to 2 at some point. The other is due to Peng and Terng [10], who investigated closed minimal hypersurfaces in a sphere the square length of whose second fundamental form is constant, thereby characterized the Cartan hypersurface in $S^{4}$.

The purpose of this paper is to give another characterization of the Cartan hypersurface in a sphere from the standpoint of Ricci tensor. In $\S 1$, we recall briefly the theory of hypersurfaces in a real space form and in $\S 2$ we outline some properties of isoparametric hypersurfaces, one of which is called a Cartan hypersurface. The Ricci tensor $S$ with components $R_{i j}$ is said to be cyclic-parallel, if the cyclic sum of the covariant

* Partially supported by JSPS and KOSEF.

** Partially supported by the Grants-in-Aid for Scientific Research, The Ministry of Education, Science and Culture, Japan 
derivatives vanishes identically, i.e., if it satisfies

$$
R_{i j k}+R_{j k i}+R_{k i j}=0,
$$

where $R_{i j k}$ denote the components of the covariant derivatives of $S$. In $\S 3$, we treat a hypersurface in a real space form whose Ricci tensor is cyclic-parallel, and in the last section it is proved that the Cartan hypersurface is the only closed hypersurface in $S^{n+1}(c)$ with constant mean curvature whose Ricci tensor is cyclic-parallel but not parallel (see Theorem 4.1).

1. Preliminaries. In order to fix notation, we briefly recall the theory of hypersurfaces in a Riemannian manifold of constant curvature. Let $\bar{M}=M^{n+1}(c)$ denote an $(n+1)$-dimensional connected Riemannian manifold of constant curvature $c$ and let $M$ denote an $n(\geqq 2)$-dimensional connected Riemannian manifold. We denote by $\phi$ a fixed isometric immersion of $M$ into $\bar{M}$. When the argument is local, $M$ need not be distinguished from $\phi(M)$. Thus, for simplicity, a point $x$ in $M$ may be identified with the point $\phi(x)$ and a tangent vector $X$ at $x$ may also be identified with the tangent vector $d \phi(X)$ at $\phi(x)$ via the differential $d \phi$ of $\phi$. We choose a local field $\left\{e_{1}, \cdots, e_{n}, e_{n+1}\right\}$ of orthonormal frames in $\bar{M}$ in such a way that, restricted to $M$, the vectors $e_{1}, \cdots, e_{n}$ are tangent to $M$ and hence the other $e_{n+1}$ is normal to $M$. Let $\left\{\bar{\omega}_{1}, \cdots, \bar{\omega}_{n}, \bar{\omega}_{n+1}\right\}$ be the field of dual frames associated with the above frame field. Throughout the present paper the following convention on the range of indices are used, unless otherwise stated:

$$
\begin{gathered}
A, B, \cdots=1,2, \cdots, n, n+1, \\
i, j, \cdots=1,2, \cdots, n .
\end{gathered}
$$

Then, the structure equations of $\bar{M}$ are given by

$$
\begin{gathered}
d \bar{\omega}_{A}+\sum_{B} \bar{\omega}_{A B} \wedge \bar{\omega}_{B}=0, \quad \bar{\omega}_{A B}+\bar{\omega}_{B A}=0, \\
d \bar{\omega}_{A B}+\sum_{C} \bar{\omega}_{A C} \wedge \bar{\omega}_{C B}=c \bar{\omega}_{A} \wedge \bar{\omega}_{B},
\end{gathered}
$$

where $\bar{\omega}_{A B}$ denote the connection forms on $\bar{M}$. The restriction of these forms $\bar{\omega}_{A}$ and $\bar{\omega}_{A B}$ to $M$ are simply denoted by $\omega_{A}$ and $\omega_{A B}$ without bar, respectively. Hence we have $\omega_{n+1}=0$. The metric on $M$ induced from the Riemannian metric $\bar{g}$ in the ambient space $\bar{M}$ by the immersion $\phi$ is given by $g=2 \sum_{i} \omega_{i} \cdot \omega_{i}$. Then $\left\{e_{1}, \cdots, e_{n}\right\}$ becomes a field of orthonormal frames on $M$ with respect to this metric and $\omega_{1}, \cdots, \omega_{n}$ are the canonical forms on $M$. It is clear from $\omega_{n+1}=0$ and the Cartan lemma that

$$
\omega_{n+1 i}=\sum_{j} h_{i j} \omega_{j}, \quad h_{i j}=h_{j i} .
$$


The quadratic form $\sum_{i, j} h_{i j} \omega_{i} \omega_{j}$ is called the second fundamental form on $M$. The $(1,1)$ tensor field $A$ on $M$ defined by

$$
g(A X, Y)=\sum_{i, j} h_{i j} \omega_{i}(X) \omega_{j}(Y)
$$

for any vector fields $X$ and $Y$ is called the shape operator of $M$. The eigenvalues $\lambda_{1}, \cdots, \lambda_{n}$ of the shape operator $A_{x}$ at each point $x$ in $M$ are called the principal curvatures at $x$ for the immersion. Furthermore, $\operatorname{Tr} A / n=\sum_{i} \lambda_{i} / n$ is called the mean curvature of $M$ at $x$. In terms of the canonical forms $\omega_{i}$ and the connection forms $\omega_{i j}$, the structure equations on the hypersurface $M$ are given as follows:

$$
\begin{gathered}
d \omega_{i}+\sum_{j} \omega_{i j} \wedge \omega_{j}=0, \quad \omega_{i j}+\omega_{j i}=0, \\
d \omega_{i j}+\sum_{k} \omega_{i k} \wedge \omega_{k j}=\Omega_{i j}, \\
\Omega_{i j}=-\sum_{k, l}\left(R_{i j k l} / 2\right) \omega_{k} \wedge \omega_{l},
\end{gathered}
$$

where $\Omega_{i j}$ (resp. $R_{i j k l}$ ) denotes the curvature form (resp. the curvature tensor) on $M$. By means of the above structure equations of $M$ and $\bar{M}$, the Gauss equation of the hypersurface is obtained as

$$
R_{i j k l}=c\left(\delta_{i l} \delta_{j k}-\delta_{i k} \delta_{j l}\right)+h_{i l} h_{j k}-h_{i k} h_{j l},
$$

and the Ricci tensor $S$ with components $R_{i j}$ and the scalar curvature $R$ can be respectively expressed as follows:

$$
\begin{gathered}
R_{i j}=(n-1) c \delta_{i j}+h h_{i j}-\sum_{r} h_{i r} h_{r j}, \\
R=n(n-1) c+h^{2}-\sum_{i, j} h_{i j} h_{i j},
\end{gathered}
$$

where $h$ is a function defined by $h=\sum_{i} h_{i i}$ so that it satisfies $h=n H$ for the mean curvature $H$ of the hypersurface.

Now, the components $h_{i j k}$ and $R_{i j k}$ of the covariant derivatives of the second fundamental form and $S$ are respectively defined by

$$
\begin{aligned}
\sum_{k} h_{i j k} \omega_{k} & =d h_{i j}-\sum_{k} h_{k j} \omega_{k i}-\sum_{k} h_{i k} \omega_{k j}, \\
\sum_{k} R_{i j k} \omega_{k} & =d R_{i j}-\sum_{k} R_{k j} \omega_{k i}-\sum_{k} R_{i k} \omega_{k j} .
\end{aligned}
$$

Since the ambient space $\bar{M}$ is of constant curvature, we get the Codazzi equation

$$
h_{i j k}-h_{i k j}=0 .
$$

Moreover, the Ricci tensor satisfies the following equation 


$$
\sum_{k} R_{i j k} \omega_{k}=\sum_{k}\left\{h h_{i j k}+h_{k} h_{i j}-\sum_{r}\left(h_{i r k k} h_{r j}+h_{i r} h_{r j k}\right)\right\} \omega_{k},
$$

where $d h=\sum_{k} h_{k} \omega_{k}$.

The Ricci tensor $S$ of a Riemannian manifold is said to be cyclicparallel if

$$
R_{i j k}+R_{j k i}+R_{k i j}=0 .
$$

From the last equation and the second Bianchi identity it is easily seen that the scalar curvature $R$ of $M$ is constant everywhere (cf. [3]). It follows from (1.12) and (1.13) that the Ricci tensor $S$ of the hypersurface $M$ is cyclic-parallel if and only if

$$
h_{k} h_{i j}+h_{j} h_{i k}+h_{i} h_{k j}+3 h h_{i j k}-2 \sum_{r}\left(h_{i r} h_{r j k}+h_{j r} h_{r k i}+h_{k r} h_{r i j}\right)=0 .
$$

REMARK 1. Riemannian manifolds with volume-preserving or, equivalently, divergence-preserving geodesic symmetries were studied by D'Atri and Nickerson [3], [4], Vanhecke [18] and so on. Such a manifold is called a D'Atri space. An analytic Riemannian manifold is known to be a D'Atri space if and only if it satisfies an infinite sequence of equations for the curvature tensor and its covariant derivatives. The cyclic-parallel condition is the first equation in the infinite sequence. The class of D'Atri spaces includes Riemannian locally symmetric spaces and harmonic spaces. For D'Atri spaces we refer the reader to [17], for instance.

REMARK 2. By [6] and [13] there exist Riemannian manifolds whose Ricci tensors are cyclic-parallel but not parallel. Gray [6] and Simon [12] independently obtained a sufficient condition for a Riemannian manifold with cyclic-parallel Ricci tensor to become an Einstein manifold or to have a parallel Ricci tensor.

2. Cartan hypersurfaces. In this section some properties of isoparametric hypersurfaces will be outlined. From now on we assume $\bar{M}=$ $M^{n+1}(c)$ in Section 1 to be complete and simply connected. Accordingly, it is a sphere $S^{n+1}(c)$, a Euclidean space $\boldsymbol{R}^{n+1}$ or a hyperbolic space $H^{n+1}(c)$. A smooth real valued function $f$ defined on an open set $U$ in $\bar{M}$ is said to be isoparametric, if $\|d f\|^{2}$ and $\bar{\Delta} f$ are functions of $f$, where $\bar{\Delta}$ denotes the Laplacian operator of $\bar{M}$. A hypersurface $M$ of $\bar{M}$ is said to be $i s o-$ parametric, if for each point $p$ of $M$, there exist an open neighborhood $U$ of $p$ in $\bar{M}$ and an isoparametric function $f$ defined on $U$ such that $U \cap M$ is a level hypersurface of $f$. On the other hand, for a connected hypersurface $M$ with a smooth unit normal $\xi$ and for a given $\varepsilon>0$, a family of parallel hypersurfaces $\phi_{t}: M \rightarrow \bar{M}$ is defined by $\phi_{t}(x)=\exp _{x} t \xi_{x}$ 
for any $t$ in $(-\varepsilon, \varepsilon)$, where exp denotes the exponential map on $\bar{M}$. As [1] showed, $\phi_{0}(M)=M$ has constant principal curvatures if and only if each $M_{t}=\phi_{t}(M)$ has constant mean curvature. Furthermore, the above property is equivalent to the fact that each $M_{t}$ has constant principal curvatures. Since a family of level hypersurfaces of an isoparametric function is parallel and each level hypersurface has constant mean curvature, the hypersurface $M$ is isoparametric if and only if $M$ has constant principal curvatures. For an isoparametric hypersurface $M$ in $\bar{M}$, let $\mu_{1}, \cdots, \mu_{k}$ with $\mu_{1}>\cdots>\mu_{k}$ be distinct principal curvatures with multiplicities $n_{1}, \cdots, n_{k}$, respectively. Then E. Cartan [1] obtained the following identities: for each $a, 1 \leqq a \leqq k$,

$$
\sum_{b \neq a} n_{b}\left(1+\mu_{b} \mu_{a}\right) /\left(\mu_{b}-\mu_{a}\right)=0 .
$$

These basic identities impose some restrictions on the range of the number of distinct principal curvatures and their multiplicities. In fact, if $c \leqq 0$, then $k \leqq 2$. In particular, if $k=2$, then $c+\mu_{1} \mu_{2}=0$. Furthermore, an $n(\geqq 2)$-dimensional complete isoparametric hypersurface $M$ with two distinct principal curvatures in $\bar{M}$ is completely classified in [1], [9] and so on, and it is isometric to one of the following spaces: $S^{p} \times S^{n-p}, S^{p} \times$ $\boldsymbol{R}^{n-p}$ or $S^{p} \times H^{n-p}$. Notice that the Ricci tensor of each of these hypersurfaces is parallel. The following theorem is due to E. Cartan [1], [2] and Münzner [8].

THEOREM 2.1. Let $M$ be a compact isoparametric hypersurface in $S^{n+1}(c)$ and let $\mu_{1}, \cdots, \mu_{k}$ with $\mu_{1}>\cdots>\mu_{k}$ be the distinct constant principal curvatures with multiplicities $n_{1}, \cdots, n_{k}$. Then the following properties are valid:

(1) $k$ is either $1,2,3,4$ or 6 .

(2) If $k=3$, then $n_{1}=n_{2}=n_{3}=2^{r}(r=0,1,2,3)$.

(3) There exists an angle $\theta \in(0, \pi / k)$ such that

$$
\mu_{a}=c^{1 / 2} \cot \{(a-1) \pi / k+\theta\}, \quad a=1, \cdots, k .
$$

The calculation of the mean curvature of each $M_{t}$ in an isoparametric family $\left\{M_{t}\right\}$ of hypersurfaces in $S^{n+1}(c)$ implies that there exists a unique minimal hypersurface in this family. In [1] and [2], E. Cartan determined all isoparametric hypersurfaces in $S^{n+1}$ with three distinct principal curvatures. In this case, each compact isoparametric hypersurface was shown to be homogeneous. In particular, the Cartan hypersurface in $S^{4}$ defined in the introduction is the only complete minimal hypersurface in $S^{4}$ with three distinct constant principal curvatures up to congruence in $S^{4}$, and others are all rigid, because each type number is greater than 4 
at any point (cf. Ryan [11]). A compact hypersurface in $S^{n+1}(c)$ having principal curvatures $(3 c)^{1 / 2}, 0$ and $-(3 c)^{1 / 2}$ with the same multiplicity $m$ $(=n / 3)$ is called a Cartan hypersurface.

For later use, we now derive a property of the Ricci tensors of Cartan hypersurfaces.

Proposition 2.2. Let $M$ be a Cartan hypersurface in $S^{n+1}(1)$. Then the Ricci tensor is cyclic-parallel but not parallel.

Proof. Since the second fundamental form $h_{i j}$ on $M$ is diagonalizable, a local field $\left\{e_{i}\right\}$ of orthonormal frames on $M$ can be chosen in such a way that $h_{i j}=\lambda_{i} \delta_{i j}$, namely,

$$
\begin{aligned}
& h_{a b}=3^{1 / 2} \delta_{a b}, \quad a, b, \cdots=1, \cdots, m, \\
& h_{r s}=0, \quad r, s, \cdots=m+1, \cdots, 2 m, \\
& h_{x y}=-3^{1 / 2} \delta_{x y}, \quad x, y, \cdots=2 m+1, \cdots, n, \\
& h_{i j}=0 \text { for other } i \text { and } j,
\end{aligned}
$$

because the principal curvatures are constant. Then the covariant derivatives $h_{i j k}$ of $h_{i j}$ satisfy

$$
\begin{gathered}
h_{a b k}=h_{r s k}=h_{x y k}=0 \text { for any } k, \\
\left\{\begin{array}{l}
\sum_{k} h_{a s k} \omega_{k}=-3^{1 / 2} \omega_{a s}, \\
\sum_{k} h_{a y k} \omega_{k}=-2 \times 3^{1 / 2} \omega_{a y}, \\
\sum_{k} h_{r y k} \omega_{k}=-3^{1 / 2} \omega_{r y} .
\end{array}\right.
\end{gathered}
$$

It follows from (2.2) that there are indices $a, s$ and $y$ such that $h_{a s y} \neq 0$, which means that the second fundamental form on $M$ is not parallel. Since, by [7], the Ricci tensor of a hypersurface in $S^{n+1}$ is parallel if and only if the second fundamental form is parallel, the Ricci tensor of the Cartan hypersurface is not parallel. On the other hand, (2.1) yields

$$
\left(\lambda_{i}+\lambda_{j}+\lambda_{k}\right) h_{i j k}=0 \text { for any } i, j \text { and } k,
$$

because $M$ has three distinct principal curvatures $3^{1 / 2}, 0$ and $-3^{1 / 2}$ with the same multiplicity $m$. This is equivalent to

$$
\sum_{r}\left(h_{i r} h_{j k r}+h_{j r} h_{k i r}+h_{k r} h_{i j r}\right)=0 .
$$

3. Case without simple roots. Let $M$ be an $n$-dimensional hypersurface in $M^{n+1}(c)$ with cyclic-parallel Ricci tensor and let $H$ be the mean curvature of $M$. In this section, we assume that $A \operatorname{grad} H=0$, namely, $\quad \sum_{j} h_{i j} h_{j}=0$. 
For the sake of brevity, a tensor $\left(h_{i j}\right)^{m}$ and a function $\alpha_{m}$ on $M$ for any integer $m$ are introduced as follows:

$$
\left\{\begin{array}{l}
\left(h_{i j}\right)^{m}=\sum_{i_{1}, \cdots, i_{m-1}} h_{i i_{1}} h_{i_{1} i_{2}} \cdots h_{i_{m-1} j}, \\
\alpha_{m}=\sum_{i}\left(h_{i i}\right)^{m}, \quad \alpha_{0}=4,
\end{array}\right.
$$

where $\alpha_{1}=h=n H$. Since the scalar curvature $R$ of $M$ is constant, so is the function $h^{2}-\alpha_{2}$, which implies

$$
d \alpha_{2}=2 h d h \text {. }
$$

The generalization of this fact is desirable. A formula we give below might be useful for this purpose. Obviously, we have

$$
d \alpha_{m}=\sum_{i, j} m\left(h_{i j}\right)^{m-1} d h_{i j},
$$

which together with (1.10) gives

$$
d \alpha_{m}=m \sum_{i, j, k} h_{i j k}\left(h_{i j}\right)^{m-1} \omega_{k},
$$

because $\left(h_{i j}\right)^{m}$ is symmetric with respect to the indices $i$ and $j$, and the connection form $\omega_{i j}$ is skew-symmetric with respect to $i$ and $j$. First of all, we have the following equation:

LEMMA 3.1. Let $M$ be an n-dimensional hypersurface in $M^{n+1}(c)$ with cyclic-parallel Ricci tensor. If $A \operatorname{grad} H=0$, then we have

$$
d \alpha_{m+1}=(m+1) \sum_{k=0}^{m}(3 h / 4)^{k} \alpha_{m-k} d h / 4
$$

for any integer $m \geqq 1$.

Proof. We show (3.5) by induction on $m$. (3.3) shows that the case where $m=1$ in (3.5) is valid, because of $\alpha_{0}=4$. Suppose that (3.5) holds when $m$ is replaced by $m-1$. Multiplying $\left(h_{i j}\right)^{m-1}$ to (1.15) and summing up the result for $j$ and $i$, we find

$$
h_{k} \alpha_{m}+3 h \sum_{i, j} h_{i j k}\left(h_{i j}\right)^{m-1}=4 \sum_{i, j} h_{i j k}\left(h_{i j}\right)^{m}+2 \sum_{i, j, h} h_{k h} h_{i j h}\left(h_{i j}\right)^{m-1}
$$

with the aid of (3.1) and (3.2), which together with (3.4) implies

$$
\alpha_{m} d h+3 h d \alpha_{m} / m=4 d \alpha_{m+1} /(m+1)+2 \sum_{i, j} h_{i j} \omega_{j} d \alpha_{m}\left(e_{i}\right) / m
$$

By (3.1) and the induction assumption, the last term on the right hand side vanishes identically and $m$ is replaced by $m+1$ in (3.5), hence

$$
4 d \alpha_{m+1}=(m+1)\left\{\alpha_{m}+3 h \sum_{k=0}^{m-1}(3 h / 4)^{k} \alpha_{m-1-k} / 4\right\} d h,
$$


which yields (3.5).

q.e.d.

We define a function $H_{m}$ for any integer $m(\geqq 2)$ by

$$
H_{m}=\sum_{k=0}^{m}(-1)^{k}\left(\begin{array}{c}
m / 4 \\
k
\end{array}\right) h^{k} \alpha_{m-k} .
$$

LEMMA 3.2. Let $M$ be a hypersurface in $M^{n+1}(c)$ with cyclic-parallel Ricci tensor. If $A$ grad $H=0$, then $H_{m}$ is constant on $M$ for any integer $m \geqq 2$.

Proof. A straightforward calculation by means of (3.5) gives

$$
d H_{m}=\sum_{k=1}^{m-1} h^{k-1} \alpha_{m-k} f(k)+h^{m-1} f(m),
$$

where a function $f$ on $\boldsymbol{Z}^{+}$is given by

$$
f(k)=\left(\frac{3}{4}\right)^{k-1} \sum_{r=0}^{k-1}(-1)^{r}\left(\begin{array}{c}
m / 4 \\
r
\end{array}\right) \frac{m-r}{4}\left(\frac{3}{4}\right)^{-r}+(-1)^{k} k\left(\begin{array}{c}
m / 4 \\
k
\end{array}\right),
$$

which can be shown to vanish by induction on $k$. Therefore $H_{m}$ is constant on $M$.

q.e.d.

The equation (3.6) is rewritten as

$$
\begin{aligned}
\alpha_{m}= & (-1)^{m-1}\left\{4\left(\begin{array}{c}
m / 4 \\
m
\end{array}\right)-\left(\begin{array}{c}
m / 4 \\
m-1
\end{array}\right)\right\} h^{m}+m h \alpha_{m-1} / 4+H_{m} \\
& -\sum_{k=2}^{m-2}(-1)^{k}\left(\begin{array}{c}
m / 4 \\
k
\end{array}\right) h^{k} \alpha_{m-k},
\end{aligned}
$$

which enables us to obtain

$$
\alpha_{m+1}-h \alpha_{m}=\sum_{k=2}^{m+1} H_{k} G_{m+1-k},
$$

where $G_{k}$ denotes a polynomial in $h$ with constant coefficients such that $\operatorname{deg} G_{k} \leqq k$. Since the second fundamental form $h_{i j}$ is diagonalizable, an orthonormal basis $\left\{e_{i}\right\}$ at a point $x$ on $M$ can be chosen in such a way that $h_{i j}=\lambda_{i} \delta_{i j}$, namely, $\lambda_{1}, \cdots, \lambda_{n}$ are the principal curvatures at the point $x$. A principal curvature $\lambda_{i}$ is called a simple root at $x$ if the multiplicity at $x$ is equal to one. We then prove:

Lemma 3.3. Let $M$ be a hypersurface in $M^{n+1}(c)$ with cyclic-parallel Ricci tensor. If the shape operator $A$ of $M$ has no simple roots on $M$, then $A \operatorname{grad} H=0$.

PRoof. For the shape operator $A$ and for a point $x$ of $M$, we denote by $E_{A}(x)$ the number of distinct eigenvalues of $A_{x}$. Let $M_{A}$ be the set 
of points $x$ such that $E_{A}$ is constant in a neighborhood of $x$. Then $M_{A}$ is clearly an open and dense subset in $M$. In each connected component of $M_{A}$, the eigenvalues of $A$ are well-defined and are distinct smooth functions everywhere so that the distribution of the spaces of eigenvectors corresponding to each eigenvalue can be defined. They are mutually orthogonal smooth distributions in each connected component of $M_{A}$. Because of the assumption that the second fundamental form $h_{i j}$ has no simple roots, there is an index $s \neq r$ in $[r]=\left\{s: \lambda_{r}=\lambda_{s}\right\}$ at each point $x$ in $M_{A}$. In this case we have $h_{r r}=h_{s s}$ in a neighborhood $U$ of $x$ in $M_{A}$ and hence

$$
h_{r r k}=h_{s s k} \text { in } U
$$

for any index $k$.

On the other hand, when $k=i=j=r$ in (1.15), we have

$$
h_{r} \lambda_{r}+\left(h-2 \lambda_{r}\right) h_{r r r}=0 \text { in } U \text {. }
$$

When $k=r, i=j=s$ in (1.15), we also have, by the Codazzi equation for the submanifold,

$$
h_{r} \lambda_{s}+3\left(h-2 \lambda_{r}\right) h_{s s r}=0 \text { in } U,
$$

and consequently $h_{r} \lambda_{r}+3\left(h-2 \lambda_{r}\right) h_{s s r}=0$. Thus it follows from (3.8) and (3.9) that $h_{r} \lambda_{r}=0$ and hence $h_{i} \lambda_{i}=0$ for any index $i$ in $U$. Accordingly, $\sum_{j} h_{i j} h_{j}=0$ on $M_{A}$ and hence on $M$, because $M_{A}$ is dense. q.e.d.

Under the same assumption as that in Lemma 3.3, we are going to prove that the mean curvature $H$ of $M$ is constant. The function $\alpha_{m}$ can be written as

$$
\alpha_{0}=4, \quad \alpha_{1}=h=\sum_{i=1}^{m} \lambda_{i}, \quad \alpha_{m}=\sum_{i=1}^{n} \lambda_{i}^{m}, \quad m=2,3, \cdots .
$$

Now, let $f_{1}(\lambda), \cdots, f_{n}(\lambda)$ be elementary symmetric functions of $\lambda=$ $\left(\lambda_{1}, \cdots, \lambda_{n}\right)$, that is,

$$
\left\{\begin{array}{l}
f_{1}=f_{1}(\lambda)=-\sum_{i} \lambda_{i}, \\
f_{2}=f_{2}(\lambda)=(-1)^{2} \sum_{i<j} \lambda_{i} \lambda_{j}, \\
\cdots \\
\quad \cdots \\
f_{n}=f_{n}(\lambda)=(-1)^{n} \prod_{i=1}^{n} \lambda_{i} .
\end{array}\right.
$$

Then it is well known that $f_{1}, \cdots, f_{n}$ and $\alpha_{1}, \cdots, \alpha_{n}, \alpha_{n+1}$ are related by the Newton formulas: 


$$
\sum_{k=0}^{m-1} f_{k} \alpha_{m-k}+m f_{m}=0 \text { for any integer } m \leqq n+1,
$$

where $f_{0}=1$ and $f_{m}=0$ for $m>n$. When these formulas are regarded as linear homogeneous simultaneous equations with respect to $\left(1, f_{1}, \cdots, f_{n}\right)$, the determinant of coefficients vanishes identically by elimination theory. By means of (3.7) and the Laplace expansion of this determinant, we can verify that $\alpha_{1}$ is the root of an algebraic equation with constant coefficients, unless all $H_{m}$ vanish. The mean curvature $H$ is then constant on $M$. But, if all $H_{m}$ 's are zero, then (3.7) says $\alpha_{m}=h \alpha_{m-1}$ for all $m \geqq 2$. Since the shape operator $A$ of $M$ is assumed to have no simple roots, $M$ is totally geodesic by a simple algebraic calculation involving (3.11) and (3.12).

Combining these facts with Lemmas 3.2 and 3.3 , one finds the following:

Proposition 3.4. Let $M$ be a hypersurface in $M^{n+1}(c)$ with cyclicparallel Ricci tensor. If the shape operator of $M$ has no simple roots, the mean curvature of $M$ is constant.

The following property follows from (3.10), Lemma 3.1 and Proposition 3.4.

COROLLARY 3.5. Let $M$ be a hypersurface in $M^{n+1}(c)$ with cyclicparallel Ricci tensor. If the shape operator of $M$ has no simple roots, then the principal curvatures of $M$ are constant.

REMARK. Let $M$ be a hypersurface in $M^{n+1}(c)$ with cyclic-parallel Ricci tensor. If the number of distinct principal curvatures is equal to two and if the multiplicity of one of them is equal to one, then they are constant and hence $M$ has a parallel Ricci tensor.

4. A characterization. We now characterize the Cartan hypersurfaces in $S^{n+1}$. A hypersurface with parallel Ricci tensor in a real space form $M^{n+1}(c)(c \neq 0)$ is completely determined in [7], for example, and the number of distinct principal curvatures of the hypersurface is at most two. Accordingly, we may assume that the Ricci tensor is not parallel. We know the following:

THEOREM 4.1. Let $M$ be a closed hypersurface in $S^{n+1}(c)$ with constant mean curvature. If the Ricci tensor $S$ of $M$ is cyclic-parallel but not parallel, then $M$ is congruent to one of the Cartan hypersurfaces.

We devote the rest of this paper to the proof of this theorem. We first bound the number of distinct principal curvatures of $M$ from above 
as follows, since we would like to conclude it to be at least three.

LEMMA 4.2. The number of distinct eigenvalues of the shape operator $A$ is at most four.

Proof. The covariant derivative $h_{i j k l}$ of $h_{i j k}$ is

$$
\sum_{l} h_{i j k l} \omega_{l}=d h_{i j k}-\sum_{l} h_{l j k} \omega_{l i}-\sum_{l} h_{i l k} \omega_{l j}-\sum_{l} h_{i j l} \omega_{l k}
$$

Then by (1.15) we have

$$
3 h h_{i j k l}=2 \sum_{r}\left(h_{i r} h_{r j k l}+h_{j r} h_{r i k l}+h_{r k} h_{r i j l}+h_{r i l} h_{r j k}+h_{r j l} h_{r i k}+h_{r l k} h_{r i j}\right) \text {. }
$$

Taking the skew-symmetric part with respect to the indices $i$ and $l$ and making use of the Ricci formula, we have

$$
3 h \sum_{r}\left(R_{l i j r} h_{r k}+R_{l i k r} h_{j r}\right)=2 \sum_{r}\left\{h_{l r} h_{r j k i}-h_{i r} h_{r j k l}+R_{l i k r}\left(h_{j r}\right)^{2}-R_{l i j r}\left(h_{r k}\right)^{2}\right\} \text {. }
$$

Summing up this equation with respect to $l$ and $k$, we get

$$
\begin{aligned}
2 \sum_{r, s} h_{r s i} h_{r s j}= & 2 \sum_{r, s}\left\{R_{r j i s}\left(h_{r s}\right)^{2}-2 R_{r j s r}\left(h_{s i}\right)^{2}+2 \sum_{r, s, l} R_{l i r s} h_{r j} h_{s l}\right. \\
& +3 h \sum_{r, s}\left(R_{r j s r} h_{s i}-R_{r j i s} h_{r s}\right),
\end{aligned}
$$

since the Ricci tensor $R_{i j}$ and the second fundamental form $h_{i j}$ commute with each other. If we substitute (1.7) and (1.8) into this last equation, then we get

$$
\begin{aligned}
2 \sum_{r, s} h_{r s i} h_{r s j}= & c\left(2 \alpha_{2}-3 h^{2}\right) \delta_{i j}+\left\{(3 n+2) c h+2 \alpha_{3}-3 h \alpha_{2}\right\} h_{i j} \\
& +\left(3 h^{2}+2 \alpha_{2}-4 n c\right)\left(h_{i j}\right)^{2}-4 h\left(h_{i j}\right)^{3} .
\end{aligned}
$$

On the other hand, by (1.7) and (1.8) and the Ricci formula for $h_{i j}$, we easily have

$$
\begin{aligned}
2 \sum_{r, s} h_{r s} h_{i j r s}= & 2\left(3 h^{2}-4 \alpha_{2}\right) \delta_{i j}+\left(3 h \alpha_{2}-4 \alpha_{3}-3 n c h\right) h_{i j} \\
& +\left(4 c n-3 h^{2}\right)\left(h_{i j}\right)^{2}+4 h\left(h_{i j}\right)^{3} .
\end{aligned}
$$

By (1.15), the covariant derivative of $\left(h_{i j}\right)^{2}$ is given by

$$
2\left(h_{i j}\right)_{k}^{2}=3 h h_{i j k}-2 \sum_{r} h_{k r} h_{r i j},
$$

which yields

$$
2\left(h_{i j}\right)_{k}^{3}=2 \sum_{r}\left(h_{i r}\right)^{2} h_{r j k}+\sum_{s}\left(3 h h_{i s k}-2 \sum_{r} h_{k r} h_{r s i}\right) h_{s j},
$$

and hence

$$
\left(h_{i j}\right)_{k}^{3}-\left(h_{i k}\right)_{j}^{3}=3 \sum_{r} h\left(h_{j r} h_{r k i}-h_{k r} h_{r j i}\right) / 2
$$


The Ricci formula for $\left(h_{i j}\right)^{3}$ together with the above equation implies that

$$
\begin{aligned}
\Delta\left(h_{i j}\right)^{3}= & 3 h\left(\sum_{r} \Delta h_{i r} h_{r j}+\sum_{r, s} h_{r s i} h_{r s j}-\sum_{r, s} h_{i j r s} h_{r s}\right) / 2-\sum_{r, s} R_{r i j s}\left(h_{r s}\right)^{3} \\
& +\sum_{r} R_{i r}\left(h_{r j}\right)^{3} .
\end{aligned}
$$

The covariant derivative of (4.4) gives

$$
\begin{aligned}
\Delta\left(h_{i j}\right)^{3}= & \sum_{r, s}\left(h_{i r}\right)_{s}^{2} h_{r s j}+\sum_{r}\left(h_{i r}\right)^{2} \Delta h_{r j}+3 h\left(\sum_{r} \Delta h_{i r} h_{r j}+\sum_{r, s} h_{r s i} h_{r s j}\right) / 2 \\
& -\sum_{r, s, t} h_{r s}\left(h_{r i t s} h_{t j}+h_{r t i} h_{s t j}\right) .
\end{aligned}
$$

By (4.1) and (4.2), the last two equations are reduced to the following relationship for the shape operator:

$$
\begin{aligned}
4 h A^{4}+ & 2\left(2 n c-3 h^{2}\right) A^{3}+3\left(3 h^{3} / 4+h \alpha_{2}-\alpha_{3}-2 n c h\right) A^{2} \\
& +\left[3 h\left(4 \alpha_{3}-3 h \alpha_{2}\right) / 4-\alpha_{4}-3 c\left\{\alpha_{2}-(3 n+4) h^{2} / 4\right\}\right] A \\
& +c\left\{3 h\left(4 \alpha_{2}-3 h^{2}\right) / 4-\alpha_{3}\right\} I=0,
\end{aligned}
$$

where $I$ denotes the identity map of the tangent space.

q.e.d.

We are now ready to prove Theorem 4.1. By the rigidity of the Cartan hypersurfaces, it suffices to show that $M$ is minimal and has three distinct principal curvatures. Since the number $k$ of distinct principal curvatures is at most four by Lemma 4.2, suppose first that $k=4$. Then (4.5) implies that $h \neq 0$. Let $\mu_{a}(a=1, \cdots, 4)$ be the distinct principal curvatures of $M$ with multiplicities $n_{a}$. Then Theorem 2.1 implies

$$
\mu_{a}=c^{1 / 2} \cot \{(a-1) \pi / 4+\theta\}, \quad 0<\theta<\pi / 4
$$

for all $a$, which yield

$$
\prod_{a} \mu_{a}=c^{2}, \quad \sum_{a<b<c} \mu_{a} \mu_{b} \mu_{c}=-c \sum_{a} \mu_{a} .
$$

Since $\mu_{a}(a=1, \cdots, 4)$ are all distinct solutions of the algebraic equation (4.5), a simple calculation for the relationship between the solutions and the coefficients gives rise to

$$
\begin{aligned}
& \alpha_{3}-h\left(3 \alpha_{2}-9 h^{2} / 4-4 c\right)=0, \\
& \alpha_{4}-3 h \alpha_{3}+3\left(3 h^{2} / 4+c\right) \alpha_{2}-3(3 n-4) c h^{2} / 4-4 n c^{2}=0 .
\end{aligned}
$$

If the term $\alpha_{4}$ is eliminated from the second equation of (4.6) and the trace of (4.5), then we have

$\left(2 h^{2}-\alpha_{2}+n c\right) \alpha_{3}+\left\{\alpha_{2}-9 h^{2} / 4-(n+4) c\right\} h \alpha_{2}+(9 n / 4-2) c h^{3}+4 n c^{2} h=0$, which is equivalent to

$$
h \sum_{i, j, k} h_{i j k} h_{i j k}=0,
$$


by virtue of (4.1) and the first equation of (4.6). This means that the second fundamental form must be parallel, because $h$ is non-zero, a contradiction to the assumption. Hence $k=3$. Let $\mu_{a}(a=1,2,3)$ be distinct principal curvatures with multiplicity $m$. For any indices $i, j$ and $k$ such that $[i],[j]$ and $[k]$ are mutually distinct, (1.15) implies

$$
(3 m-2) h h_{i j k}=0 \text {. }
$$

Thus $h$ must vanish, namely, the hypersurface $M$ is minimal. Then, by (1.15), the trace of (4.5) and the minimality of $M$, we have $\alpha_{3}\left(\alpha_{2}-n c\right)=0$, hence $\alpha_{3}$ vanishes identically. Thus the equation (4.5) implies

$$
\mu_{1}=\lambda>0, \quad \mu_{2}=0 \text { and } \mu_{3}=-\lambda,
$$

with $\lambda^{2}=\left(\alpha_{4}+3 c \alpha_{2}\right) / 4 n c$, and hence

$$
\lambda^{2}=3 c
$$

because they are all of the same multiplicity. Since the Cartan hypersurfaces are the only closed minimal hypersurfaces in $S^{n+1}$ with three distinct principal curvatures up to congruence in $S^{n+1}$, the proof of Theorem 4.1 is complete.

\section{BIBLIOGRAPHY}

[1] E. CARTAN, Familles de surfaces isoparamétriques dans les espaces à courbure constante, Ann. di Mat. 17 (1938), 177-191.

[2] E. CARTAN, Sur des familles remarquables d'hypersurfaces isoparamétriques dans les espaces sphériques, Math. Zeit. 45 (1939), 335-367.

[3] J. E. D'Atri AND H. K. Nickerson, Divergence-preserving geodesic symmetries, J. Differential Geometry 3 (1969), 467-476.

[4] J.E. D'Atri AND H. K. NiCKerson, Geodesic symmetries in spaces with special curvature tensors, J. Differential Geometry 9 (1974), 251-262.

[5] S. Kobayashi, Compact homogeneous hypersurfaces, Trans. Amer. Math. Soc. 88 (1958), 137-143.

[6] A. Gray, Einstein-like manifolds which are not Einstein, Geometriae Dedicata 7 (1978), $259-280$.

[7] I. Mogi and H. NAKagawa, On hypersurfaces with parallel Ricci tensor in a Riemannian manifold of constant curvature, Differential Geometry in honor of $\mathrm{K}$. Yano, Kinokuniya, Tokyo, 1972, 267-279.

[8] H.F. MÜNZNER, Isoparametrische Hyperflächen in Sphären I and II, Math. Ann. 251 (1980), 57-71; ibid. 256 (1981), 215-232.

[9] H. OZEKI AND M. TAKEUCHI, On some types of isoparametric hypersurfaces in spheres I and II, Tôhoku Math. J. 27 (1975), 515-559; ibid. 28 (1976), 7-55.

[10] C. K. Peng and C. L. Terng, Minimal hypersurfaces of spheres with constant scalar curvature, Seminar on minimal submanifolds, Ann. of Math. Studies, 103, Princeton Univ. Press, 1983, 177-205.

[11] P.J. RYAN, Homogeneity and some curvature conditions for hypersurfaces, Tôhoku Math. J. 21 (1969), 363-388. 
[12] U. Simon, On differential operators of second order on Riemannian manifolds with nonpositive curvature, Colloq. Math. 31 (1974), 223-229.

[13] T. Suмiтомо, On a certain class of Riemannian homogeneous spaces, Colloq. Math. 26 (1972), 129-133.

[14] R. TAKAgI, Homogeneous hypersurfaces in a sphere with the type number 2, Tôhoku Math. J. 23 (1971), 49-58.

[15] R. TAKAGI AND T. TAKAHASHI, On the principal curvature on homogeneous hypersurfaces in a sphere, Differential Geometry in honor of K. Yano, Kinokuniya, Tokyo, 1972, 469-481.

[16] T. TAKAHASHI, Homogeneous hypersurfaces in spaces of constant curvature, J. Math. Soc. Japan 22 (1970), 395-410.

[17] F. TRICERRI AND L. VANHECKE, Homogeneous structures on Riemannian manifolds, London Math. Soc. Lecture Notes 83, 1983.

[18] L. VANHECKe, Some solved and unsolved problems about harmonic and commutative spaces, Bull. Soc. Math. Belg. 34 (1982), 1-24.

KYUNGPOOK UNIVERSITY AND UNIVERSITY OF TSUKUBA

TAEGU, 635

KOREA

IBARAKI, 305

JAPAN 